\title{
Provision of acute and elective general surgical care at a tertiary facility in the era of subspecialisation
}

\author{
J H Klopper, MMed (Surg); S Rayamajhi, FCS (SA); J J Venter, MB ChB, H Dip Surg (SA); D J de Villiers, FCS (SA), MMed (Surg); \\ N Almgla, MB ChB; J C Kloppers, Dip PEC (SA), MRCS (Ed), FCS (SA), MMed (Surg), FRCS (Gen Surg) (Ed), Cert Gastro (SA)
}

Department of Surgery, Groote Schuur Hospital and Faculty of Health Sciences, University of Cape Town, South Africa

Corresponding author: J H Klopper (juan.klopper@uct.c.za)

\begin{abstract}
Background. The need for an acute care and general surgical unit (ACGSU) to provide care for patients previously managed on an ad hoc basis by subspecialist units was recognised by the provincial government of the Western Cape Province, South Africa, the management of Groote Schuur Hospital (GSH) and the Department of Surgery.

Objective. To describe the resulting ACGSU and its functioning.

Methods. Data available from administrative records, patient files and operating room forms were collected in spreadsheet form for the period July 2013 - November 2016 inclusive.

Results. The ACGSU comprised a medical care team of four consultants and four to five trainees. A total of 7571 patients were seen during the study period, the majority (66.1\%) referred from the GSH Emergency Centre. Skin and soft-tissue infections formed the major disease complex. A total of 3144 operative records were available. The most common procedures were wound debridement and inguinal hernia repairs. Trainees acted as primary surgeon in most cases. Complications (Clavien-Dindo grades I - V) were noted in $25.0 \%$ of patients.

Conclusions. The ACGSU provides patient management that would otherwise complicate care in the subspecialist surgical units. It serves as a training ground for registrars and stands as a model for other institutions. Further research into the effect on patient care is planned.
\end{abstract}

S Afr Med J 2017;107(11):948-951. DOI:10.7196/SAMJ.2017.v107i11.12335

The duty of treating surgical patients in need of urgent care has historically fallen to the members of different surgical units, such as hepatopancreaticobiliary, gastrointestinal and vascular surgery. ${ }^{[1]}$ This model ultimately required surgeons with little experience or interest in conditions outside their subspecialty to treat emergency cases. Surgery has become increasingly subspecialised as a result of the rapid expansion of medical knowledge and technologies, an increase in patient demand and a variety of personal factors. ${ }^{[1,2]}$ The trauma care model introduced in the late 1960s showed the benefits of restricting the management of injured patients to a dedicated team and subsequently led to the development of trauma surgery as a subspecialty, with many units established throughout the world. ${ }^{[3,4]}$

An acute care and general surgery unit (ACGSU) similarly isolates surgical patients requiring urgent care and provides a dedicated team of surgeons for their management. ${ }^{[5,6]}$ This model has already been shown to improve outcome. ${ }^{[7-11]}$ It frees other units to concentrate on elective care, provides an alternative career path to trauma surgeons in developed-world settings, and potentially allows for planning of the optimal use of resources. ${ }^{[12,13]}$

South African (SA) tertiary institutions have not been immune to the changes in the surgical landscape. The Division of General Surgery at Groote Schuur Hospital (GSH), Cape Town, consists of well-established subspecialty units with recognised fellowship training. Concerns regarding the management of emergency, nontrauma surgical conditions and general surgical care were recognised by the provincial government and the institution in 2010. The proposed solution was the establishment of a unit that could respond to the local demand and infrastructure.

\section{Objective}

To describe the academic ACGSU that resulted from the initial decision to separate the care of the above cases from the subspecialist units and to describe its functioning in a tertiary surgical setting in SA, so as to offer an example for other institutions with similar demands.

\section{Methods}

Retrospective data collected from July 2013 to November 2016 were analysed. As the ACGSU was a new surgical unit, no such collection existed prior to this period. Data were captured from patient files, as well as ward and operating room administrative records.

Selected variables were collected in spreadsheet format, and statistical analysis of the data available for the study period was performed using the iPython Jupyter notebook kernel ${ }^{[14]}$ of the Python programming language with the following additional libraries: numerical Python, ${ }^{[15]}$ scientific Python, Statsmodels and Scikit-learn. Data were managed by the Pandas library. ${ }^{[14-19]}$

Descriptive statistics included point estimates and measures of dispersion. Comparison of numerical variables was conducted by appropriate parametric and non-parametric tests. Categorical variables were analysed using the $\chi^{2}$ test for independence and nonparametric tests if ordinal.

A two-tail test hypothesis was used with an alpha value of 0.05 as discriminator for rejection of null hypotheses.

\section{Resulits}

\section{Clinical services}

Patient demographics are listed in Table 1. The main function of the ACGSU is the management of surgical conditions requiring 
urgent attention. The process is initiated at first consultation. These consultations mainly take place in the acute admissions area of the GSH Emergency Centre, but the clinical service extends to all inpatients in GSH, and consultation may take place in the ward or unit in which such patients are present. Elective cases are seen in the weekly clinic as first contact. A total of 7571 patients were referred during the study period, and the referring facilities are listed in Table 2.

The ACGSU also provides an emergency outreach programme to the tertiary care obstetrics and gynaecology unit at Mowbray Maternity Hospital, where most consultations take place during surgery. It is the norm for such patients to be transferred to GSH postoperatively, if appropriate.

Administrative admission records were available for 2483 patients. The logistics of initial admission are listed in Table 3, and the most common admission diagnoses in Table 4.

The ACGSU has a minor procedure theatre list for cases performed under local anaesthesia. Typical procedures include biopsies for diagnosis of skin lesions, lymph node excisions, and muscle and nerve biopsies. In addition to this there is a general anaesthesia abscess list three times a week where the referring doctor can book cases directly.

The ACGSU also provides an emergency upper and lower gastrointestinal endoscopy service, with endoscopies performed in

Table 1. Patient demographics $(N=7571)$

\begin{tabular}{lll}
\hline Characteristic & Female & Male \\
\hline Count, $n(\%)$ & $4028(53.2)$ & $3543(46.8)$ \\
Mean age (years) & 51 & 50
\end{tabular}

Table 2. Referring facilities $(N=7571)$

\begin{tabular}{ll}
\hline Referral unit & $\boldsymbol{n}(\%)$ \\
\hline Emergency Centre & $5003(66.1)$ \\
Other units in Groote Schuur Hospital & $1263(16.7)$ \\
Surrounding hospitals & $803(10.6)$ \\
Smaller facilities in Cape Town & $334(4.4)$ \\
Other & $168(2.2)$
\end{tabular}

Table 3. Admission logistics $(N=2483)$

\begin{tabular}{ll}
\hline Place of admission & $\boldsymbol{n}(\%)$ \\
\hline Direct admission to ACGSU ward & $1959(78.9)$ \\
Emergency surgery prior to admission & $415(16.7)$ \\
Direct admission to the critical care unit & $109(4.4)$ \\
ACGSU = acute care and general surgical unit. &
\end{tabular}

Table 4. Most common emergency surgical diagnoses $(N=2$ 483)

\begin{tabular}{ll}
\hline Condition & $\boldsymbol{n}$ (\%) \\
\hline Non-diabetic skin and soft-tissue infections & $611(24.6)$ \\
Diabetic skin and soft-tissue infections & $541(21.4)$ \\
Acute appendicitis & $213(8.6)$ \\
Acute cholecystitis & $124(5.0)$ \\
Bowel obstruction (all causes) & $121(4.9)$
\end{tabular}

the endoscopy suite, at the bedside in critical care units, and in the operating theatre as required.

The secondary function of the ACGSU extends to a set of elective procedures. As GSH serves as the primary care facility for the nearby suburbs, general surgical procedures required by this population are performed on two full-day, elective theatre lists. These two lists also provide access to emergency procedures deferred for later attention, such as cholecystectomies presenting $>96$ hours after onset of symptoms. These patients are booked as elective cases, while still falling under the care of the ACGSU.

At discharge, patients are scheduled to attend a weekly clinic if required.

All team members share patient management, and formal ward rounds are held twice a day. Patients are not assigned to individual consultants or registrars, so a formal handover round is held every morning and every weekday afternoon to inform all members of the status of and care for each patient.

All cases considered to require consultation with other units and departments at GSH are discussed by personal communication with the relevant personnel, with folder notes identifying required information.

\section{Surgical procedures}

The ACGSU has access to the 24-hour theatre facilities at GSH. In addition, a semi-elective overflow list is available on Tuesdays. All emergency cases are formally logged on the booking system of the operating room complex.

Case complexity is identified by a system of colour coding applied by the ACGSU surgeon, comprising green, yellow, orange and red cases. All cases except those earmarked as green are discussed with the anaesthetist involved, and based on this individual discussion, colour assignment may be altered.

Red cases are considered extremely ill with marked physiological decompensation. Common underlying causes include sepsis and haemorrhage. Despite aggressive resuscitative measures, these patients require immediate surgical care. Orange cases require surgery within an hour for conditions such as arterial flow compromise including limbs and bowel, hollow-organ perforations, and varying degrees of necrotising fasciitis. Yellow cases require surgery within 6 hours and are patients who are physiologically stable despite a serious surgical diagnosis, including peritonitis. Green cases are those in which a reasonable delay would not alter the outcome, yet surgical intervention is required during the current admission.

Surgical procedure records were available for 3144 patients, 1644 $(52.3 \%)$ of whom were female. The median age was 46 (range 13 $100)$ years for females and 51 (range 13 - 92) years for males $(p<0.01)$.

A consultant surgeon acted as primary surgeon in 526 cases $(16.7 \%)$ and a trainee in $2618(83.3 \%)$. In the case of elective work, the ratio of consultant $v$. registrar as primary surgeon was $146 / 758$ $(16.2 \%$ v. $83.8 \%)$. In the case of emergency surgery, this ratio was $180 / 2060(8.4 \%$ v. $91.6 \%)(p<0.01)$.

Overall, consultants assisted registrars in 768 cases. In the case of elective surgery, a consultant assisted a registrar in 552 cases (61.6\%), with a more junior doctor assisting in the rest of the cases. During emergency procedures, a more junior team member acted as first assistant to a registrar in 2068 cases (90.2\%), as opposed to 193 cases in which a consultant was the first assistant $(p<0.01)$.

When a consultant surgeon acted as primary surgeon, the mean (standard deviation (SD)) duration of the surgical procedure was 88.3 (62.7) minutes, and when a trainee acted as primary surgeon, the mean duration was 52.3 (37.1) minutes $(p<0.01)$.

A total of 2248 procedures (71.5\%) were classified as emergencies, while $896(28.5 \%)$ were elective. Of the emergency procedures, 220 
(9.8\%) were coded as green, $1485(66.1 \%)$ as yellow, $506(22.5 \%)$ as orange and $37(1.6 \%)$ as red.

The most common procedures are listed in Table 5.

The majority of cases ( $n=2377,75.6 \%$ ) were performed under general anaesthesia. A total of 601 cases (19.1\%) were performed under regional anaesthesia.

A total of 2785 patients (88.6\%) were returned to the ward after the procedure. The remaining 359 (11.4\%) were admitted to the critical care unit.

The Clavien-Dindo classification for postoperative complications ${ }^{[20]}$ was available in the last 2 months of the study period (Table 6). A total of 209 deaths (2.8\% of all patients referred to the unit) were recorded over the study period. The causes of death are listed in Table 7.

\section{Discussion}

The ACGSU described above functions under the auspices of the secondary-level services of the Metro West District of Cape Town, under the administrative management of a principal specialist.

The staffing of the ACGSU was determined by the posts made available by the provincial government of the Western Cape and by the logistics of the rotational programme that must comply with the requirements of the Health Professions Council of South Africa.

Four full-time consultants provide the clinical service. The consultant core comprises two senior consultants, two junior consultants and a senior medical officer.

The unit provides tertiary education to a group of four or five registrars per 3-month rotation. They provide full daytime cover for

Table 5. Most common surgical procedures $(N=2487)$

\begin{tabular}{ll}
\hline Procedure & $\boldsymbol{n}(\%)$ \\
\hline Minor debridement & $358(11.4)$ \\
Inguinofemoral hernia repair & $352(11.2)$ \\
Laparoscopic cholecystectomy & $308(9.8)$ \\
Ventral hernia repair & $308(9.8)$ \\
Laparoscopic appendicectomy & $192(6.1)$ \\
Above-knee amputation & $160(5.1)$ \\
Open appendicectomy & $154(4.9)$ \\
Incision and drainage of abscess & $153(4.9)$ \\
Diagnostic laparotomy & $141(4.5)$ \\
Major debridement for necrotising fasciitis & $129(4.1)$ \\
Partial colectomy & $116(3.7)$ \\
Below-knee amputation & $116(3.7)$
\end{tabular}

Table 6. Clavien-Dindo postoperative classification ${ }^{[20]}$ $(N=216)$

\begin{tabular}{ll}
\hline Classification & $\boldsymbol{n}(\%)$ \\
\hline No complications & $162(75.0)$ \\
Grade I & $17(7.9)$ \\
Grade II & $15(6.9)$ \\
Grade IIIa & $7(3.2)$ \\
Grade IIIb & $6(2.8)$ \\
Grade IVa & $4(1.9)$ \\
Grade IVb & $1(0.4)$ \\
Grade V & $4(1.9)$
\end{tabular}

Table 7. Deaths $(N=209)$

\begin{tabular}{ll}
\hline Cause & $\boldsymbol{n}(\%)$ \\
\hline Inevitable (course of illness) & $155(74.2)$ \\
Other & $17(8.1)$ \\
Therapeutic error & $11(5.3)$ \\
System failure & $8(3.8)$ \\
Therapeutic error by referring facility & $6(2.9)$ \\
Diagnostic error by referring facility & $5(2.4)$ \\
Diagnostic error & $3(1.4)$ \\
Technical error & $3(1.4)$ \\
Technical error by referring facility & $1(0.5)$
\end{tabular}

all clinical work, as well as after-hours on-site cover. All registrars in the Division of General Surgery (DGS) are required to complete two 3-month rotations through the ACGSU during their 4-year training. All registrars are available for daytime duties, which include ward rounds, ward work, consultations, clinic attendance, and surgery. A single registrar provides on-site after-hours cover. A comprehensive list of surgical procedures is recorded for each registrar. It is used in their formal evaluation and to compile a portfolio required by the College of Surgery of South Africa as entry criteria for the final fellowship examination. Together with a research component, trainees can seek specialist registration with the Health Professions Council of South Africa.

The data show that the ACGSU at GSH provides both a clinical service and a broad-based training ground for future surgeons tasked with the care of a diverse population group.

The majority of patients (82.8\%) referred to the unit were from the Emergency Centre and other units in GSH. Most of these patients were admitted to the ACGSU ward. Approximately one in five, however, required direct transfer to either the operating theatre or the critical care unit.

The greatest proportion of the surgical work performed by the ACGSU is emergency in nature, relieving the subspecialist units from the burden of care, as intended by the initial decision to establish this unit. The elective work is smaller in number than the emergency work, because the geographical area of referral for secondary-level surgical work is smaller than the geographical area for referral of emergency cases. This is exacerbated by the fact that the ACGSU serves as tertiary referral centre for emergency surgical care from the surrounding hospitals, which in turn serve their own communities as far as elective surgical work is concerned.

Registrars are provided with ample opportunity to act as primary surgeon in a mix of elective and emergency procedures. They are well supported by consultants for elective cases. This assistance is considerably less for emergency cases.

\section{Conclusions}

The ACGSU at GSH plays and will play a leading academic role in surgical service delivery and provides technical experience for trainees. It has been shown to be a useful adjunct to the other subspecialist units in the DGS, freeing both their time and resources by taking away their emergency surgical workload.

Parameters highlighted during data collection can be of vital importance in planning future healthcare strategies based on the needs of various institutions and countries.

Future research should elucidate the effect on patient care in the setting of the ACGSU by way of outcome measurement. 
Acknowledgements. None.

Author contributions. All the authors contributed equally to the writing of the original article and to the data collection. JHK created the original research project and data collection tool, performed the statistical analysis and completed the revisions.

\section{Funding. None.}

\section{Conflicts of interest. None.}

1. Malangoni MA. Acute care surgery: The general surgeon's perspective. Surgery 2007;141(3):324-326 https://doi.org/10.1016/j.surg.2007.01.008

2. Stitzenberg KB, Sheldon GF. Progressive specialization within general surgery: Adding to the complexity of workforce planning. J Am Coll Surg 2005;201(6):925-932. https://doi.org/10.1016/j. complexity of workforce

3. Blaisdell FW. Development of the city-county (public) hospital. Arch Surg 1994;129(7):760-764. Blaisdell FW. Development of the city-county (public)
https://doi.org/10.1001/archsurg.1994.01420310092017

4. MacKenzie EJ, Rivara FP, Jurkovich GJ, et al. A national evaluation of the effect of trauma-center care on mortality. N Engl J Med 2006;354(4):366-378. https://doi.org/10.1056/NEJMsa052049

5. Spain DA, Miller FB. Education and training of the future trauma surgeon in acute care surgery Trauma, critical care, and emergency surgery. Am J Surg 2005;190(2):212-217. https://doi. org/10.1016/j.amjsurg.2005.05.014

6. Hoyt DB, Kim HD, Barrios C. Acute care surgery: A new training and practice model in the United States. World J Surg 2008;32(8):1630-1635. https://doi.org/10.1007/s00268-008-9576-y

7. Cubas RF, Gomez NR, Rodriguez S, Wanis M, Sivanandrum A, Garberoglio CA. Outcomes in the management of appendicitis and cholecystitis in the setting of a new acute care surgery service model. Impact on timing and cost. JACS 2012;215(5):715-721. https://doi.org/10.1016/j. amcollsurg.2012.06.415

8. Von Conrady D, Hamza S, Weber D, et al. The acute surgical unit: Improving emergency care. Aust $\mathrm{N}$ Z J Surg 2010;80(12):933-936. https://doi.org/10.1111/j.1445-2197.2010.05490.x
9. Lau B, Difronzo LA. An acute care surgery model improves timeliness of care and reduces hospital stay for patients with acute cholecystitis. Am Surg 2011;77(10):1318-1321.

10. Gandy RC, Truskett PG, Wong SW, et al. Outcomes of appendicectomy in an acute care surgery model. Med J Aust 2010;193(5):281-284.
Man

11. Shaina S, Dirks R, Davis J. Comparison of outcomes of patients with acute appendicitis between an Shaina S, Dirks R, Davis J. Comparison of outcomes of patients with acute appendicitis between an
acute care surgery model and traditional call coverage model in the same community. Am J Surg 2016;212(6):1083-1089. https://doi.org/10.1016/j.amjsurg.2016.09.006

12. Gamelli RL. Organization of faculty practice and resident training in acute care surgery in an academic medical center. Surgery 2007;141(3):302-303. https://doi.org/10.1016/j.surg.2007.01.005

13. Trydestam C, Pratto S, Cushing B, Whiting J. Effect of a dedicated acute care operating room on hospital efficiency. Am Surg 2014;80(1):89-91.

14. Pérez F, Granger BE. IPython: A system for interactive scientific computing. Comput Sci Eng 2007;9(3):21-29. https://doi.org/10.1109/MCSE.2007.53

15. Van der Walt S, Colbert C, Varoquaux G. The NumPy array: A structure for efficient numerical computation. Comput Sci Eng 2011;13(2):22-30. https://doi.org/10.1109/MCSE.2011.37

16. Kubilius J. A framework for streamlining research workflow in neuroscience and psychology. Front Neuroinform 2014;7. https://doi.org/10.3389/fninf.2013.00052

17. Pedregosa F, Varoquaux G, Gramfort A, et al. Scikit-learn: Machine learning in Python. J Mach Learn Res 2011;12(10):2825-2830. http://www.jmlr.org/papers/volume12/pedregosalla/pedregosalla.pdf (accessed 4 October 2017).

18. McKinney W. Data structures for statistical computing in Python, Proceedings of the 9th Python in Science Conference, Austin, Texas, 28 June - 3 July 2010. http://conference.scipy.org/proceedings/ scipy2010/mckinney.html (accessed 25 September 2017).

19. Hunter JD. Matplotlib: A 2D graphics environment. Comput Sci Eng 2007;9(3):90-95. https://doi. org/10.1109/MCSE.2007.55

20. Dindo D, Demartines N, Clavien PA. Classification of surgical complication: A new proposal with evaluation in a cohort of 6336 patients and results of a survey. Ann Surg 2004;240(2):205-213. https:// doi.org/10.1097/01.sla.0000133083.54934.ae

Accepted 24 May 2017. 